\title{
The preliminary ethnobotanical study of medicinal plants in Uşak (Turkey)
}

\author{
Gizem Bulut, M. Zahid Bozkurt, Ertan Tuzlacı
}

\begin{abstract}
This study was made to reveal the plants used as traditional folk medicine in Uşak in 2013. The specimens of the plants used as folk remedies have been collected and the information about the local names, the part(s) used, the ailments treated, the therapeutic effect, the preparation, the methods of administration, and the duration of treatment were recorded. The plant specimens are kept in the Herbarium of the Faculty
\end{abstract}

of Pharmacy, Marmara University. As a result of identification of the plant specimens, 38 species, used as a traditional folk medicine in Uşak, have been determined. According to the majority of the plants which have similar usages, the plants were mostly used for gastrointestinal system diseases, respiratory system diseases and urinary system diseases.

Key Words: Ethnobotany; Folk medicinal plants; Uşak; Turkey.
Gizem Bulut, Ertan Tuzlac1

Department of Pharmaceutical Botany, Faculty of Pharmacy, Marmara University, İstanbul, Turkey

M. Zahid Bozkurt

Lokman Eczanesi, No:3/A, Uşak

Corresponding Author:

Gizem Bulut

e-mail: gizem.bulut@marmara.edu.tr

Submitted / Gönderilme: 10.11.2016 Revised/ Düzeltme: 01.12.2016

\section{Introduction}

Nowadays around the world, treatment with traditional folk medicine still maintains its importance especially when modern health services become inadequate. Almost $80 \%$ of general population in the world use plants to treat several illnesses (1). Turkey is located in a region where the influences of many civilizations can be seen in terms historical background. That's why, it has a rich traditional culture (2). Traditional folk medicine creates an important part of this culture. At the same time, our country has a rich flora. In West Anatolia where our research area is situated in, a lot of ethnobotanical researches (3-33) were conducted and a study related to Uşak was found out.

Uşak is a city in the interior part of the Aegean Region in Turkey (Fig. 1). The city has a population of 187.886 (2012) and it is the central district of Uşak Province. The city was previously known by its Greek name of Ousakeion. It is bordered by Kütahya to the north, Afyon to the east, Denizli to the south and Manisa to the west. Northern and eastern parts of Uşak is surrounded by mountains, and there are lowlands and rolling lands in the southern and western parts of the city. Mount Elma is located in the north of central district. 
There are wide tablelands and grasslands on the mountain reaching up to $1805 \mathrm{~m}$. Another important elevation points of the city are Mount Ahır (1915 m), Tahtalı Hill (1644 m) and Kocatepe (1298) (http://www.yerelnet.org.tr/ the last accessed date 10.10.2016).

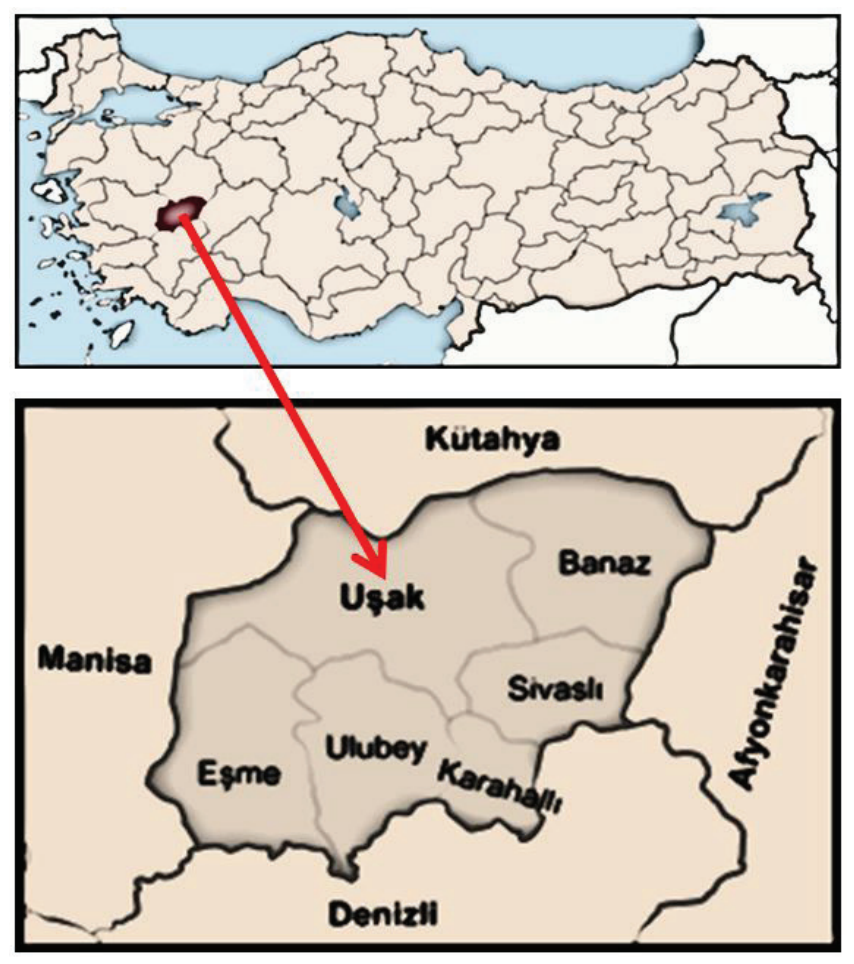

Figure 1: Map of Uşak

\section{Material and methods}

Ethnobotanical data was collected by means of open and semi-structured interviews (34-36) with local people. The interviews took the form of general conversations and a strict questionnaire (Appendix). We asked the participants to show us methods for preparing the medicinal plants that they used, and we usually collected the plants with local people. In some cases, certain plants were collected beforehand from the same locality that the interviews were made.

During the interviews, various information was collected (e.g. local names, part(s) of the plants used, ailments treated, therapeutic effect, methods of preparation and methods of administration) from experienced adults and patients. In addition, any harmful effects of the folk-medicine, if declared, were also recorded. The plant specimens are kept in the Herbarium of the Faculty of Pharmacy, Marmara University (MARE). The "Flora of Turkey and the East Aegean Islands" $(37,38)$ were mainly used for the identification of the plants.

\section{Results and discussion}

The plants used for medicinal purposes in Uşak are presented in Table 1 alphabetically arranged according to their botanical names with the relevant information. Taxonomical changes according to the Plant List (http://www. theplantlist. org/ the last accessed date 08.11.2016) are shown in square brackets in Table 1 together with their popular scientific names. Thirty-eight medicinal plant species, belonging to 25 families, were recorded in the research area. Of these, 26 species were wild, and 12 species were cultivated plants. The most common medicinal plant families were Lamiaceae (15\%) and Rosaceae (10\%).

During the study, a total of 51 remedies were recorded. Most remedies were taken internally (90\%).

The plant parts most commonly used for the preparation of remedies were leaves $(25.5 \%)$, aerial parts $(21.6 \%)$ and fruits (15.6\%).

The main preparation methods were infusion and decoction. According to the interviewees, Ecballium elaterium, should be handled with care since an over-dose could prove dangerous. We compared our results with other comprehensive ethnobotanical studies on folk medicinal plants which have already been carried out in the neighbouring areas $(2,4,5$, 16-18, 20, 21, 32, 33) and presented in Table 1. According to this table, Rosa canina recorded in eight localities is the most common herbal medicinal plant in Ușak and its surroundings.

According to the majority of the plants which have similar usage, the plants are mostly used for gastrointestinal system diseases, respiratory system diseases and urinary system diseases.

Some of medicinal plants have been recorded as wild edible plants in our study area. They include: Malva neglecta, Crataegus szovitsii, Cydonia oblonga, Juglans regia, Rumex crispus and Urtica urens. Peganum harmala is used to not only medicinal but also amulet which is very common usages. In addition, Herniaria hirsuta L. (MARE 15018) is used as soap in Uşak (Fig. 2).
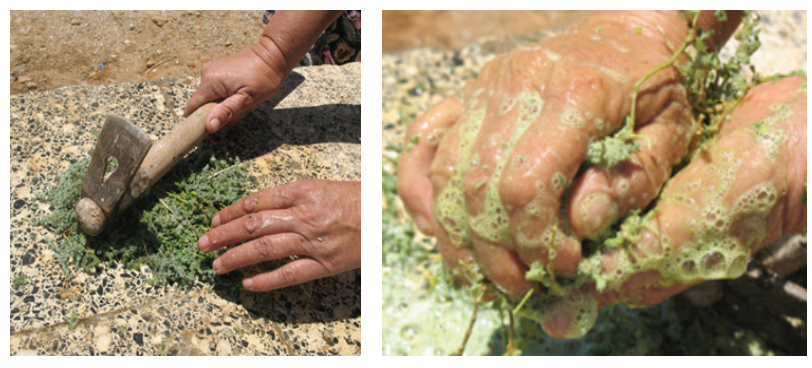

Figure 2: Hernaria hirsuta used as soap 
Table 1 Folk medicinal plants of Uşak (Turkey)

\begin{tabular}{|c|c|c|c|c|c|}
\hline $\begin{array}{l}\text { Botanical name, family and specimen } \\
\text { number }\end{array}$ & Local name & $\begin{array}{l}\text { Plant part } \\
\text { used }\end{array}$ & $\begin{array}{l}\text { Ailments treated/ } \\
\text { Therapeutic effect }\end{array}$ & $\begin{array}{l}\text { Preparations/ } \\
\text { Administration }\end{array}$ & $\begin{array}{l}\text { Similar usage in } \\
\text { literature }\end{array}$ \\
\hline $\begin{array}{l}\text { Alcea rosea } \mathrm{L}^{\mathrm{a}} \\
\text { (Malvaceae, MARE } 15023 \text { ) }\end{array}$ & $\begin{array}{l}\text { Peygamber } \\
\text { övenderesi } \\
\end{array}$ & Flowers & Kidney stones & Infusion, int. & $(21)^{b}$ \\
\hline $\begin{array}{l}\text { Amygdalus communis L. }{ }^{a} \\
\text { [Prunus dulcis (Mill.)D.A.Webb.] } \\
\text { (Rosaceae, MARE 15025) }\end{array}$ & Acı badem & Seeds & Diabetes & Crushed, int. & Diabetes (20) \\
\hline $\begin{array}{l}\text { Anethum graveolens L. }{ }^{\text {a }} \\
\text { (Apiaceae, MARE 15005) }\end{array}$ & Dere otu & Aerial parts & Stomach diseases & Cooked, eaten & $(20)^{b}$ \\
\hline $\begin{array}{l}\text { Avena sterilis L. subsp. } \text { sterilis }^{\text {a }} \\
\text { (Poaceae, MARE 14994) }\end{array}$ & Yulaf & Aerial parts & Rheumatism & Crushed, ext. & \\
\hline $\begin{array}{l}\text { Capparis ovata Desf. } \\
\text { [Capparis spinosa L.] } \\
\text { (Capparaceae, MARE 15020) }\end{array}$ & Kapari & Fruits & Rheumatism & - , eaten & $(2,18)^{b}$ \\
\hline $\begin{array}{l}\text { Carduus nutans L. } \\
\text { (Asteraceae, MARE 14990) }\end{array}$ & Gül dikeni & Seeds & Liver diseases & Crushed then infusion, int. & $(21)^{b}$ \\
\hline $\begin{array}{l}\text { Convolvulus arvensis L. } \\
\text { (Convolvulaceae, MARE 14991) }\end{array}$ & Tosbağa otu & \begin{tabular}{|l|}
$\begin{array}{l}\text { Flowers \& } \\
\text { leaves }\end{array}$ \\
\end{tabular} & Wound & Crushed (+flour), ext. & $(2,17,18,21)^{b}$ \\
\hline $\begin{array}{l}\text { Crataegus szovitsii Pojark. } \\
\text { [Crataegus orientalis Pall. ex M.Bieb. } \\
\text { subsp. szovitsii (Pojark.) K.L. Chr.] } \\
\text { (Rosaceae, MARE 15006) }\end{array}$ & Alıç & $\begin{array}{l}\text { Flowers \& } \\
\text { leaves }\end{array}$ & $\begin{array}{l}\text { Cardiovascular system } \\
\text { diseases }\end{array}$ & Infusion, int. & \\
\hline $\begin{array}{l}\text { Cupressus sempervirens } \mathrm{L}^{\mathrm{a}} \\
\text { (Cupressaceae, MARE 15008) }\end{array}$ & Selvi kozalağı & Cones & Shortness of breath & Decoction, int. & $(20,21)^{b}$ \\
\hline $\begin{array}{l}\text { Cydonia oblonga Miller } \\
\text { (Rosaceae, MARE 15012) }\end{array}$ & Ayva & $\begin{array}{l}\text { Leaves } \\
\text { Leaves } \\
\text { Fruits }\end{array}$ & $\begin{array}{l}\text { Cough } \\
\text { Stomach diseases } \\
\text { Cardiovascular system } \\
\text { diseases }\end{array}$ & $\begin{array}{l}\text { Decoction, int. } \\
\text { Decoction, int. } \\
\text {-, eaten }\end{array}$ & $\begin{array}{l}\text { Cough }(2, \\
16,17)^{b}\end{array}$ \\
\hline $\begin{array}{l}\text { Cynodon dactylon (L.) Pers. var. } \\
\text { villosus Regel } \\
\text { (Poaceae, MARE 14986) }\end{array}$ & Ayrık kökü & $\begin{array}{l}\text { Roots } \\
\text { Roots }\end{array}$ & $\begin{array}{l}\text { Diuretic } \\
\text { Kidney stones }\end{array}$ & $\begin{array}{l}\text { Decoction, int. } \\
\text { Decoction, int. }\end{array}$ & $\begin{array}{l}\text { Kidney stones } \\
(16,17,18)\end{array}$ \\
\hline $\begin{array}{l}\text { Ecballium elaterium (L.)A. Rich. } \\
\text { (Cucurbitaceae, MARE 15014) }\end{array}$ & Acr bostan & $\begin{array}{l}\text { Roots } \\
\text { Fruits juice }\end{array}$ & $\begin{array}{l}\text { Hemorrhoids } \\
\text { Sinusitis }\end{array}$ & $\begin{array}{l}\text { Grated, int. } \\
\text { Dropped into the nostrils }\end{array}$ & $\begin{array}{l}\text { Hemorrhoids }(17) \\
\text { Sinusitis }(2,4,18,20 \text {, } \\
21,33)\end{array}$ \\
\hline $\begin{array}{l}\text { Elaeagnus angustifolia L. }{ }^{\text {a }} \\
\text { (Elaeagnaceae, MARE 14996) }\end{array}$ & İğde & Leaves & Urinary system diseases & Decoction, int. & $\begin{array}{l}\text { Urinary system } \\
\text { diseases }(16,20,32) \\
(18)^{\mathrm{b}}\end{array}$ \\
\hline $\begin{array}{l}\text { Equisetum ramosissimum Desf. } \\
\text { (Equisetaceae, MARE 15000) }\end{array}$ & Kurkkilit otu & \begin{tabular}{|l} 
Aerial parts \\
Aerial parts \\
\end{tabular} & $\begin{array}{l}\text { Kidney Stones } \\
\text { Rheumatism } \\
\end{array}$ & $\begin{array}{l}\text { Infusion, int. } \\
\text { Infusion, int. }\end{array}$ & $(2,5,18)^{b}$ \\
\hline $\begin{array}{l}\text { Hyoscyamus niger } \mathrm{L} . \\
\text { (Solanaceae, MARE 15015) }\end{array}$ & Kulak otu & Seeds & Earache & $\begin{array}{l}\text { Seeds are spread on dying } \\
\text { embers and ears are exposed } \\
\text { to fum, ext. }\end{array}$ & $\begin{array}{l}\text { Earache }(4,32) \\
(20)^{\mathrm{b}}\end{array}$ \\
\hline $\begin{array}{l}\text { Hypericum perforatum L. } \\
\text { (Hypericaceae, MARE 14997) }\end{array}$ & Sarı kantaron & $\begin{array}{l}\text { Flowering } \\
\text { branches } \\
\text { Flowering } \\
\text { branches } \\
\end{array}$ & $\begin{array}{l}\text { Sedative } \\
\text { Stomach diseases }\end{array}$ & $\begin{array}{l}\text { Infusion, int. } \\
\text { Infusion, int. }\end{array}$ & $\begin{array}{l}\text { Stomach diseases } \\
(2,4,5,18,20)\end{array}$ \\
\hline $\begin{array}{l}\text { Juglans regia L. } \\
\text { (Juglandaceae, MARE 15003) }\end{array}$ & Ceviz & Seeds & $\begin{array}{l}\text { Cardiovascular system } \\
\text { diseases }\end{array}$ & $\begin{array}{l}\text { Kept into the water for one } \\
\text { night, int. }\end{array}$ & $\begin{array}{l}\text { Cardiovascular } \\
\text { system diseases }(20) \\
(2,4,5,16,17,32)^{\mathrm{b}}\end{array}$ \\
\hline $\begin{array}{l}\text { Lactuca serriola L. } \\
\text { (Asteraceae, MARE 15022) }\end{array}$ & Eşek marulu & $\begin{array}{l}\text { Leaves } \\
\text { Leaves }\end{array}$ & $\begin{array}{l}\text { Hemorrhoids } \\
\text { Cardiovascular system } \\
\text { diseases }\end{array}$ & $\begin{array}{l}\text { Infusion, int. } \\
\text { Infusion, int. }\end{array}$ & $(5,18)^{\mathrm{b}}$ \\
\hline $\begin{array}{l}\text { Malva neglecta Wallr. } \\
\text { (Malvaceae, MARE 14988) }\end{array}$ & Ebe gümeci & $\begin{array}{l}\text { Leaves } \\
\text { Leaves }\end{array}$ & $\begin{array}{l}\text { Cough } \\
\text { Abdominal pain }\end{array}$ & $\begin{array}{l}\text { Maseration into the water for } \\
\text { one night, int. } \\
\text { Crushed, wrapped in a cloth, } \\
\text { ext. }\end{array}$ & $\begin{array}{l}\text { Abdominal pain } \\
(2,32) \\
(17,18,20)^{\mathrm{b}}\end{array}$ \\
\hline
\end{tabular}




\begin{tabular}{|c|c|c|c|c|c|}
\hline $\begin{array}{l}\text { Botanical name, family and specimen } \\
\text { number }\end{array}$ & Local name & $\begin{array}{l}\text { Plant part } \\
\text { used }\end{array}$ & $\begin{array}{l}\text { Ailments treated/ } \\
\text { Therapeutic effect }\end{array}$ & $\begin{array}{l}\text { Preparations/ } \\
\text { Administration }\end{array}$ & $\begin{array}{l}\text { Similar usage in } \\
\text { literature }\end{array}$ \\
\hline $\begin{array}{l}\text { Mentha longifolia (L.) Hudson subsp. } \\
\text { typhoides (Briq.)Harley var. typhoides } \\
\text { (Lamiaceae, MARE 14998) }\end{array}$ & Su nanesi & Aerial parts & Sprain & $\begin{array}{l}\text { Crushed (+onion, tarhana), } \\
\text { wrapped in a cloth, ext. }\end{array}$ & $(2,5,18,21,32)^{b}$ \\
\hline $\begin{array}{l}\text { Mentha x piperita L. }{ }^{a} \\
\text { (Lamiaceae, MARE 15004) }\end{array}$ & Nane & $\begin{array}{l}\text { Leaves } \\
\text { Leaves }\end{array}$ & $\begin{array}{l}\text { Cold } \\
\text { Sore throat }\end{array}$ & $\begin{array}{l}\text { Decoction, int. } \\
\text { Decoction, int. }\end{array}$ & $\begin{array}{l}\text { Cold (16) } \\
(20)^{\mathrm{b}}\end{array}$ \\
\hline $\begin{array}{l}\text { Morus alba } \text { L. }^{\mathrm{a}} \\
\text { (Moraceae, MARE 15013) }\end{array}$ & Dut & $\begin{array}{l}\text { Fruits } \\
\text { Fruits } \\
\end{array}$ & $\begin{array}{l}\text { Anaemia } \\
\text { Constipation }\end{array}$ & $\begin{array}{l}- \text {, eaten } \\
- \text {, eaten }\end{array}$ & $(18)^{\mathrm{b}}$ \\
\hline $\begin{array}{l}\text { Olea europaea L. var. europaea }{ }^{\text {a }} \\
\text { (Oleaceae, MARE 15010) }\end{array}$ & Zeytin & $\begin{array}{l}\text { Leaves } \\
\text { Leaves }\end{array}$ & $\begin{array}{l}\text { Diabetes } \\
\text { Antihypertensive }\end{array}$ & $\begin{array}{l}\text { Decoction, int. } \\
\text { Decoction, int. }\end{array}$ & $\begin{array}{l}\text { Diabetes }(2,20) \\
\text { Antihypertensive } \\
(2,20)\end{array}$ \\
\hline $\begin{array}{l}\text { Papaver rhoeas L. } \\
\text { (Papaveraceae, MARE 15017) }\end{array}$ & Gelincik & $\begin{array}{l}\text { Flowers } \\
\text { Flowers }\end{array}$ & $\begin{array}{l}\text { Cold } \\
\text { Expectorant }\end{array}$ & $\begin{array}{l}\text { Infusion, int. } \\
\text { Infusion, int. }\end{array}$ & $\begin{array}{l}\text { Expectorant }(4,16) \\
(18,33)^{\mathrm{b}}\end{array}$ \\
\hline $\begin{array}{l}\text { Peganum harmala L. } \\
\text { (Zygophyllaceae, MARE 15021) }\end{array}$ & Üzerlik & $\begin{array}{l}\text { Seeds } \\
\text { Seeds }\end{array}$ & $\begin{array}{l}\text { Eye diseases } \\
\text { Rheumatism }\end{array}$ & \begin{tabular}{|l|} 
Seeds are spread on dying \\
embers then added water, ext. \\
Seeds are spread on dying \\
embers then added water, ext.
\end{tabular} & $\begin{array}{l}\text { Rheumatism }(17) \\
(2,18,21,32)^{b}\end{array}$ \\
\hline $\begin{array}{l}\text { Plumbago europaea L. } \\
\text { (Plumbaginaceae, MARE 14993) }\end{array}$ & Soyulgan otu & Leaves & Eczema & $\begin{array}{l}\text { Crushed (+Lawsonia } \\
\text { inermis), ext. }\end{array}$ & \\
\hline $\begin{array}{l}\text { Rosa canina L. } \\
\text { (Rosaceae, MARE 14989) }\end{array}$ & Öküzgötü & Fruits & Cold & Decoction, int. & $\begin{array}{l}\text { Cold }(2,4,20,21) \\
(5,17,18,32)^{\mathrm{b}}\end{array}$ \\
\hline $\begin{array}{l}\text { Rosmarinus officinalis } \mathrm{L}^{\mathrm{a}} \\
\text { (Lamiaceae, MARE 15011) }\end{array}$ & Biberiye & $\begin{array}{l}\text { Aerial parts } \\
\text { Aerial parts }\end{array}$ & $\begin{array}{l}\text { Sedative } \\
\text { Stomach diseases }\end{array}$ & $\begin{array}{l}\text { Infusion, int. } \\
\text { Infusion, int. }\end{array}$ & Stomach diseases (20) \\
\hline $\begin{array}{l}\text { Rumex crispus L. } \\
\text { (Polygonaceae, MARE 15016) }\end{array}$ & İlabada & $\begin{array}{l}\text { Fruits } \\
\text { Fruits }\end{array}$ & $\begin{array}{l}\text { Urinary system diseases } \\
\text { Diuretic }\end{array}$ & $\begin{array}{l}\text { Decoction, int. } \\
\text { Decoction, int. }\end{array}$ & $(5,17,21)^{\mathrm{b}}$ \\
\hline $\begin{array}{l}\text { Salvia tomentosa Miller } \\
\text { (Lamiaceae, MARE 14992) }\end{array}$ & Şabla & Aerial parts & Sore throat & Infusion, int. & \begin{tabular}{|l|} 
Sore throat $(20,21)$ \\
$(5,16,17,18,32)^{\mathrm{b}}$
\end{tabular} \\
\hline $\begin{array}{l}\text { Teucrium polium L. } \\
\text { (Lamiaceae, MARE 15001) }\end{array}$ & Topalan otu & $\begin{array}{l}\text { Aerial parts } \\
\text { Aerial parts }\end{array}$ & $\begin{array}{l}\text { Stomach diseases } \\
\text { Abdominal pain }\end{array}$ & $\begin{array}{l}\text { Infusion, int. } \\
\text { Infusion, int. }\end{array}$ & $\begin{array}{l}\text { Abdominal pain } \\
(18,32) \\
(2,5)^{\mathrm{b}}\end{array}$ \\
\hline $\begin{array}{l}\text { Thymbra spicata L. var. spicata } \\
\text { (Lamiaceae, MARE 15007) }\end{array}$ & Kekik & $\begin{array}{l}\text { Aerial parts } \\
\text { Aerial parts }\end{array}$ & $\begin{array}{l}\text { Stomach diseases } \\
\text { Respiratory system } \\
\text { diseases }\end{array}$ & $\begin{array}{l}\text { Infusion, int. } \\
\text { Infusion, int. }\end{array}$ & $\begin{array}{l}\text { Respiratory system } \\
\text { diseases }(20) \\
(2,21)^{\mathrm{b}}\end{array}$ \\
\hline $\begin{array}{l}\text { Tilia platyphyllos Scop. }{ }^{\text {a }} \\
\text { (Tiliaceae, MARE 15024) }\end{array}$ & Ihlamur & $\begin{array}{l}\text { Flowers and } \\
\text { bracts } \\
\text { Flowers and } \\
\text { bracts }\end{array}$ & $\begin{array}{l}\text { Cold } \\
\text { Sedative }\end{array}$ & $\begin{array}{l}\text { Infusion, int. } \\
\text { Decoction, int. }\end{array}$ & Cold (2) \\
\hline $\begin{array}{l}\text { Tribulus terrestris L. } \\
\text { (Zygophyllaceae, MARE 15019) }\end{array}$ & Demir pitırağ & Roots & Stomach diseases & Decoction, int. & $\begin{array}{l}\text { Stomach diseases (21) } \\
(2,18,20)^{\mathrm{b}}\end{array}$ \\
\hline $\begin{array}{l}\text { Urtica urens L. } \\
\text { (Urticaceae, MARE 14987) }\end{array}$ & Isırgan & Leaves & $\begin{array}{l}\text { Respiratory system } \\
\text { diseases }\end{array}$ & Infusion, int. & $\begin{array}{l}\text { Respiratory system } \\
\text { diseases }(32) \\
(2,5,20)^{\mathrm{b}}\end{array}$ \\
\hline $\begin{array}{l}\text { Verbascum lasianthum Boiss. ex } \\
\text { Bentham } \\
\text { (Scrophulariaceae, MARE 14985) }\end{array}$ & Siğgırkuyruğu & Flowers & Hemorrhoids & $\begin{array}{l}\text { Infusion (+Viola sp. ), int., for } \\
1 \text { month }\end{array}$ & \\
\hline $\begin{array}{l}\text { Vicia faba } \text { L. }^{\mathrm{a}} \\
\text { (Fabaceae, MARE 14995) }\end{array}$ & Bakla & Leaves & Eye diseasess & -, eaten & $(20,21)^{b}$ \\
\hline $\begin{array}{l}\text { Viscum album L. subsp. album } \\
\text { [Viscum album L.] } \\
\text { (Loranthaceae, MARE 14999) }\end{array}$ & Buç, Ökse otu & $\begin{array}{l}\text { Whole plant } \\
\text { Whole plant }\end{array}$ & $\begin{array}{l}\text { Diabetes } \\
\text { Antihypertensive }\end{array}$ & $\begin{array}{l}\text { Maseration into the water, } \\
\text { int. } \\
\text { Maseration into the water, } \\
\text { int. }\end{array}$ & $\begin{array}{l}\text { Diabetes }(17,18) \\
(2,16)^{\mathrm{b}}\end{array}$ \\
\hline
\end{tabular}

Int.; Internal use. Ext.; External use. ${ }^{a}$ Cultivated plant ${ }^{\mathrm{b}}$ Different usage. 


\section{Acknowledgments}

The authors wish to thank interview participants generously sharing their knowledge about traditional medicine.

\section{Appendix 1}

\section{Questionnaire Form}

1. Name and surname of the participant.

2. Age and sex of the participant.

3. Telephone and address of the participant.

4. Educational level of the participant.

5. Date of interview.

6. Place of residence of the participant.

Uşak yöresinin tıbbi bitkileri hakkında etnobotanik ön bir araştırma

\section{ÖZ}

Bu çalışma, Uşak yöresinde 2013 yılında geleneksel halk ilacı olarak kullanılan bitkilerini saptamak amacıyla yapılmıştır. Halk ilacı olarak kullanılan bitki örnekleri toplanmış; bitkilerin yöresel adları, kullanılan kısımları, tedavideki kullanılışları, tedavideki etkisi, hazırlanışı, uygulama yöntemi
7. Duration of residence of the participant.

8. Local name of the plant.

9. Human health or Animal health.

10. Ailments treated/therapeutic effect.

11. Plant part used.

12. Preparation.

13. Administration.

14. Dosage.

15. Duration of treatment.

16. Age group of patients (baby, child, adult).

17. Side effects.

18. Different ethnobotanical use.

ve tedavi süresi ile ilgili bilgiler kayıt edilmiştir. Bitki örnekleri Marmara Üniversitesi Eczacllı Fakültesi Herbaryumu'nda saklanmaktadır. Bitki örneklerinin teşhisleri sonucunda, 38 türün Uşak'da geleneksel halk ilacı olarak kullanıldığı saptanmıştır. Görüşme yapılan kişilerin çoğunluğuna göre, bitkiler en çok mide-barsak sistemi şikayetlerinde, solunum yolları ve üriner sistem rahatsızlklarında kullanılmaktadır.

Anahtar Kelimeler: Etnobotanik; Tibbi bitkiler; Uşak; Türkiye.

\section{References}

1. IUCN, WHO, WWF. The International Union for Conservation of Nature and Natural Resources (IUCN), Gland, Switzerland, in partnership with The World Health Organization (WHO), Geneva, Switzerland, and World Wide Fund for Nature (WWF), Gland, Switzerland, 1993.

2. Bulut G, Tuzlaci E. An ethnobotanical study of medicinal plants in Turgutlu (Manisa-Turkey). J Ethnopharmacol 2013; 149: 633-47.

3. Akaydin G, Simsek I, Arituluk ZC, Yesilada E. An ethnobotanical survey in selected towns of the Mediterranean subregion (Turkey). Turk J Biol 2013; 37:230-47.

4. Akcicek E, Vural M. Kumalar dağı (Afyon) ve çevresindeki bazı bitkilerin yöresel adları ve etnobotanik özellikleri. Herb J Syst Bot 2003; 10: 151-62.

5. Arı S, Temel M, Kargığlu M, Konuk M. Ethnobotanical survey of plants used in Afyonkarahisar-Turkey. J Ethnobiol Ethnomed 2015;11:1-15.

6. Aslan A, Mat A, Ozhatay N, Sarıyar G. A contribution to traditional medicine in west Anatolia. İstanbul Ecz Fak Derg 2007; 39: 73-84.

7. Bulut G, Tuzlaci E. Bozcaada’nin Çiçekleri ve Yararli Bitkileri. Bozcaada Kaymakamlığı, İstanbul. 2009.
8. Bulut G. Folk medicinal plants of Silivri (İstanbul-Turkey). Marmara Pharm J 2011; 15: 25-9.

9. Bulut G, Tuzlaci E. An ethnobotanical study of medicinal plants in Bayramiç (Çanakkale-Turkey). Marmara Pharm J. 2015; 19: 269-82.

10. Bulut G. Medicinal and wild food plants of Marmara Island (Balikesir-Turkey). Acta Soc Bot Pol 2016; 85:3501-16.

11. Duran A, Satıl F, Tumen G. Balıkesir Yöresinde Yenen Yabani Meyveler ve Etnobotanik Özellikleri. Herb J Syst Bot 2001; 8: 87-94.

12. Durmuskahya C, Ozturk M. Ethnobotanical survey of medicinal plants used for the treatment of diabetes in Manisa, Turkey. Sains Malays 2013; 42, 1431-8.

13. Gurdal B, Kultur S. Anethnobotanical study of medicinal plants in Marmaris (Mugla, Turkey). J Ethnopharmacol 2013; 146: 113-26.

14. Honda G, Yesilada E, Tabata M, Sezik E, Fujita T, Takeda Y, Takaishi Y, Tanaka T. Traditional Medicine in Turkey VI. Folk medicine in west Anatolia: Afyon, Kütahya, Denizli, Muğla, Aydın provinces. J Ethnopharmacol 1996; 53: 75-87.

15. Işık S, Gönüz A, Arslan Ü, Ozturk M. Afyon (Türkiye) ilindeki bazı türlerin etnobotanik özellikleri. Herb J Syst Bot 1995; 2: 161-6. 
16. Kahraman A, Tatlı A. Umurbaba dağ (Eşme-Ușak) ve çevresindeki bazı bitkilerin mahalli adları ve etnobotanik özellikleri. Herb J Syst Bot 2004; 11: 147-54.

17. Kargioglu M, Cenkci S, Serteser A, Evliyaoğlu N, Konuk M, Kok MS, Bağci Y. An ethnobotanical survey of inner-west Anatolia, Turkey. Hum Ecol 2008; 36:763-77.

18. Kargioglu M, Cenkci S, Serteser A, Evliyaoğlu N, Konuk M, Kok MS, Bağci Y. Traditional uses of wild plants in the middle Aegean region of Turkey. Hum Ecol 2010; 38:429-50.

19. Polat R, Satil F. An ethnobotanical survey of medicinal plants in Edremit Gulf (Balikesir-Turkey). J Ethnopharmacol 2012; 139: 626-41.

20. Sargin SA, Akcicek E, Selvi S. An ethnobotanical study of medicinal plants used by the local people of Alaşehir (Manisa) inTurkey. J Ethnopharm 2013; 150: 860-74.

21. Sargin SA, Selvi S, López V. Ethnomedicinal plants of Sarigöl district (Manisa), Turkey. J Ethnopharmacol 2015; 171: 64-84.

22. Sayar A, Guvensen A, Ozdemir F, Ozturk M. Muğla (Türkiye) ilindeki bazı türlerin etnobotanik özellikleri. Herb J Syst Bot 1995; 2: 151-60.

23. Uysal I, Gücel S, Tütenocakli T, Öztürk M. Studies on the medicinal plants of Ayvacık-Çanakkale in Turkey. Pakistan J Bot 2012; 44: 239-44.

24. Tuzlaci E. Honaz Dağının bitkileri II. J Fac Pharm İstanbul 1977; 13: 47-61.

25. Tuzlaci E, Aymaz PE. Turkish folk medicinal plants, part IV: Gönen (Balikesir). Fitoterapia 2001; 72: 323-43.

26. Tuzlaci E. Bodrum'da Bitkiler veYașam. Güzel Sanatlar Matbaasi, İstanbul. 2005.
27. Tuzlaci E, Sadikoğlu E. Turkish folk medicinal plants, part VI: Koçarli (Aydin). J Fac Pharm İstanbul 2007; 39: 25-37.

28. Tuzlaci E, Emre Bulut G. Turkish folk medicinal plants, Part VII: Ezine (Çanakkale). J Fac Pharm İstanbul 2007; 39: 39-51.

29. Ugulu İ, Baslar S, Yorek N, Dogan Y. The investigation and quantative ethnobotanical evaluation of medicinal plants used around İzmir province, Turkey. J Med Plants Res 2009; 3: 34567.

30. Ugulu E, Secmen Ö. Medicinal plants populary used in the villages of Yunt Mountain (Manisa-Turkey). Fitoterapia 2008; 79: 126-31.

31. Uysal I, Onar S, Karabacak E, Celik S. Ethnobotanical aspects of Kapıdağ Peninsula (Turkey). Biol Divers Cons 2010; 3: 1522.

32. Yeşilada E, Sezik E., Honda G, Takaishi Y, Takeda Y, Tanaka T. Traditional medicine in Turkey IX. Folk medicine in northwest Anatolia. J Ethnopharmacol 1999; 64: 195-210.

33. Yucel E. Tulukoglu A. Plants used as folk medicine in and around Gediz (Kütahya). Ekoloji 2000; 9: 12-4.

34. Alexiades MN. Selected Guidelines for Ethnobotanical Research: A Field Manual. The New York Botanical Garden, New York. 1996.

35. Cotton CM. Ethnobotany: Principles and Applications. John Wiley and Sons Ltd., West Sussex. 1996.

36. Martin GJ. Ethnobotany: A Methods Manual. Chapman and Hall, London. 1995.

37. Davis PH. The Flora of Turkey and the East Agean Islands. Edinburgh University Press, Edinburgh. 1965-1985.

38. Davis PH, Mill RR, Tan K. The Flora of Turkey and the East Aegean Islands. Edinburgh University Press, Edinburgh. 1988. 